\title{
EXPERIMENTAL AND NUMERICAL INVESTIGATION OF THE TURBULENT WAKE FLOW OF A GENERIC SPACE LAUNCHER CONFIGURATION
}

\section{Statnikov ${ }^{1}$, D. Saile ${ }^{2}$, J.-H. Meiß ${ }^{1}$, A. Henckels ${ }^{2}$, M. Meinke ${ }^{1}$, A. Gülhan ${ }^{2}$, and W. Schröder ${ }^{1}$}

${ }^{1}$ Institute of Aerodynamics RWTH Aachen University 5a Wüllnerstraße, Aachen 52062, Germany

${ }^{2}$ German Aerospace Center (DLR) Institute of Aerodynamics and Flow Technology Linder Höhe, Köln 51147, Germany

The turbulent wake of a generic space launcher at cold hypersonic freestream conditions is investigated experimentally and numerically to gain detailed insight into the intricate base flow phenomena of space vehicles at upper stages of the flight trajectory. The experiments are done at $\mathrm{Ma}_{\infty}=6$ and $\operatorname{Re}_{D}=1.7 \cdot 10^{6} \mathrm{~m}^{-1}$ by the German Aerospace Center (DLR) and the corresponding computations are performed by the Institute of Aerodynamics Aachen using a zonal Reynolds-averaged NavierStokes / Large-Eddy Simulation (RANS/LES) approach. Two different aft-body geometries consisting of a blunt base and an attached cylindrical nozzle dummy are considered. It is found that the wind tunnel model support attached to the upper side of the main body has a nonnegligible impact on the wake along the whole circumference, albeit on the opposite side, the effects are minimal compared to an axisymmetric configuration. In the blunt-base case, the turbulent supersonic boundary layer undergoes a strong aftexpansion on the model shoulder leading to the formation of a confined low-pressure $\left(p / p_{\infty} \approx 0.2\right)$ recirculation region. Adding a nozzle dummy causes the shear layer to reattach on the its wall at $x / D \sim 0.6$ and the base pressure level to increase $\left(p / p_{\infty} \approx 0.25\right)$ compared to the blunt-base case. For both configurations, the pressure fluctuations on the base wall feature dominant frequencies at $\mathrm{Sr}_{D} \approx 0.05$ and $\mathrm{Sr}_{D} \approx 0.2-0.27$, but are of small amplitudes $\left(p_{\mathrm{rms}} / p_{\infty}=0.02-0.025\right)$ compared to the main body boundary layer. For the nozzle dummy configuration, when moving downstream along the nozzle extension, the wall pressure is increasingly influenced by the reattaching shear layer and the periodic low-frequency behavior

This is an Open Access article distributed under the terms of the Creative Commons Attribution License 4.0, which permits unrestricted use, distribution, and reproduction in any medium, provided the original work is properly cited. 
becomes less pronounced. Directly behind the reattachment point, the wall pressure reaches maximum mean and root-mean-square (rms) values of about $p / p_{\infty}=1$ and $p_{\text {rms }}^{\prime} / p_{\infty}=0.1$ and features a broadband spectrum without distinct frequencies determined by the incoming turbulent supersonic boundary layer.

\section{NOMENCLATURE}

D main body diameter $(108 \mathrm{~mm})$

Ma Mach number

$p \quad$ local static pressure

$p^{\prime} \quad$ local static pressure fluctuation

$\operatorname{Re}_{D} \quad$ Reynolds number based on the main body diameter, $\varrho_{\infty} U_{\infty} D / \mu_{\infty}$

$\mathrm{Sr}_{D} \quad$ Strouhal number based on the main body diameter, $f D / U_{\infty}$

$X \quad$ streamwise distance (0 in the base plane)

$\varphi \quad$ azimuthal position ( $0^{\circ}$ at the strut support position)

\section{Subscripts}

rms root-mean-square

$\infty \quad$ free-stream conditions

\section{INTRODUCTION}

The wake flow of space launchers is determined by different intricate phenomena, such as flow separation at the base shoulder, reattachment of the shear layer on the outer nozzle wall, interaction with the jet plume, to name a few. Therefore, an accurate prediction of the wake flow still poses a great challenge in developing future rocket-like launch vehicles. It is generally known that the base drag of axial cylindrical bodies, which is caused by the low-pressure recirculation area at the base, constitutes a major part of the overall drag. Rollstin [1], for instance, determines the base drag of projectiles caused by the separation of the outer flow to be up to $35 \%$ of the overall drag, which can be even higher for launch vehicles due to their larger base area. Moreover, the involved base flow phenomena possess a pronounced unsteady behavior and the resulting base pressure oscillations might excite structure vibrations of critical amplitudes. Besides this aeroelastic aspect, convection of the hot gases from the jet upstream to the base area can lead to confined hot spots and thermal loads of the structure. Therefore, it is of fundamental importance to provide accurate analyses of the not yet fully understood static and dynamic behavior of the base flow for the design and optimization of reliable future space launcher systems. 
Numerous investigations of the separated base flow of space launchers have been performed in the past experimentally and numerically. On the experimental side, worth of mentioning are the experiments by Mathur and Dutton [2], who investigated an axisymmetric configuration at $\mathrm{Ma}_{\infty}=2.46$, by Bannink et al. [3], and by Scarano et al. [4] who examined an axisymmetric rocket configuration at $\mathrm{Ma}_{\infty}=2$ and 3 with an underexpanded Mach 4 nozzle flow in the context of the FESTIP (Future European Space Transportation Investigations Programme) research program.

Numerical investigations of the turbulent wakes range from various RANS model based solutions $[5,6]$ via detached-eddy simulations (DES) $[7,8]$ and LES [9] to direct numerical simulations (DNS) [10,11]. The base flow and the base pressure, however, have not always been predicted with sufficient accuracy. The RANS models were found to be suitable only for the prediction of the attached flow and failed to provide the accurate results concerning the lowpressure recirculation area behind the base. Direct numerical simulation is at the present time restricted to small Reynolds numbers and also a small integration domain. In contrast, hybrid approaches like DES [12] and zonal RANS/LES [13] allow time-resolved computation of the dynamic wake flow at practically relevant Reynolds numbers and were found to be a good compromise between costs and accuracy for time-resolved simultations of the dynamic wake flow of space launchers.

In this work, a zonal RANS/LES approach is applied on the numerical side allowing accurate time-resolved computations of the intricate wake flow field at high spatial and temporal resolution at a fraction of the costs of a pure LES. The experiments are done using high-speed measurement techniques and provide, foremost, statistically reliable data for spectral analysis of the strongly turbulent wake flow fields thanks to the wind tunnel runs with durations of several seconds. The presented investigations are performed within the framework of the German Transregional Collaborative Research Center TRR 40 founded by the German Research Foundation which focuses on the analysis and modeling of coupled liquid rocket propulsion systems and their integration into the space transportation system.

\section{GEOMETRY AND FLOW CONDITIONS}

The outer geometry of the investigated space launcher configuration is approached by a generic model which consists of a rounded conical top with an apex angle of $36^{\circ}$ attached to a cylindrical main body part with a diameter of $D=108 \mathrm{~mm}$. To analyze the influence of the nozzle extension on the base flow field, two different aft-body geometries consisting of a blunt base and an attached cylindrical nozzle dummy are considered. The diameter of the attached nozzle dummy is $d_{\text {nozzle }}=43 \mathrm{~mm}$ and the length is $l_{\text {nozzle }}=129.6 \mathrm{~mm}$ 

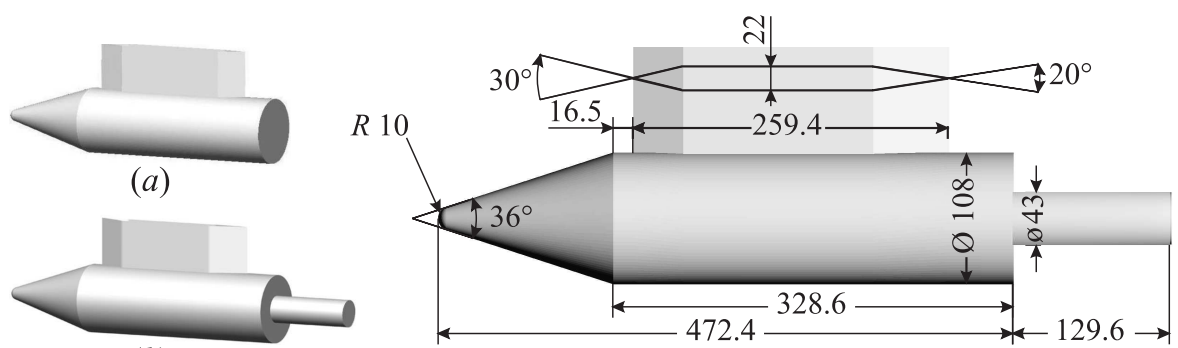

(b)

Figure 1 Geometrical parameters of the investigated generic rocket configurations. Dimensions are in millimeters

leading to the same nozzle - main body ratio of $d_{\text {nozzle }} / D_{\text {main body }} \approx 0.4$ and $l_{\text {nozzle }} / D_{\text {main body }} \approx 1.2$ as for the main stage of Ariane $\mathrm{V}$ launcher. Due to the necessity to mount the models in the wind tunnel and to enclose cables for the electronics in the base and nozzle dummy, a vertical double-wedge profiled strut is orthogonally attached to the upper side of the main body. An overview of the configurations and the geometry parameters is presented in Fig. 1.

As a baseline for the investigations, an assumed trajectory stage of an Ariane V-like space launcher [14] is defined simulating the launch conditions at an altitude of $50 \mathrm{~km}$, which corresponds to a free-stream Mach number of 6.0 and a unit Reynolds number of $17 \cdot 10^{6} \mathrm{~m}^{-1}$. In this flow regime, the fluidstructure interaction is less significant, while unsteady shock phenomena in the base region become more important. The used free-stream conditions follow the experimental setup at the DLR Cologne and are summarized in Table 1. Regarding the chosen similarity conditions, it can be stated that the shear layer expansion and subsequent recompression, which, as will be shown in section 5.1, plays the dominant role in the wake at $\mathrm{Ma}_{\infty}=6$, are considered similar to a real flight. Former investigations, e.g., by Murthy [15], have shown that the Reynolds number has little influence on the base pressure/pressure ratio as long as the incoming boundary layer is turbulent, which is the case for the current flow problem as was shown by Saile et al. [16]. The shear layer expansion is, mainly, a function of the Mach number. The unsteady effects in the wake flow are expected to scale with the main body diameter and the free-stream velocity; that is why, the Strouhal number $\operatorname{Sr}_{D}=f D / U_{\infty}$ is used to quantify characteristic frequencies of the unsteady wake flow.

Table 1 Free-stream conditions

\begin{tabular}{ccccccc}
\hline $\mathrm{Ma}$ & $\mathrm{Re}, 1 / \mathrm{m}$ & $U, \mathrm{~m} / \mathrm{s}$ & $p_{0}, \mathrm{~Pa}$ & $p, \mathrm{~Pa}$ & $T_{0}, \mathrm{~K}$ & $T, \mathrm{~K}$ \\
\hline 6.0 & $16 \cdot 10^{6}$ & 910 & $18 \cdot 10^{5}$ & $1.14 \cdot 10^{3}$ & 470 & 57 \\
\hline
\end{tabular}




\section{EXPERIMENTAL SETUP}

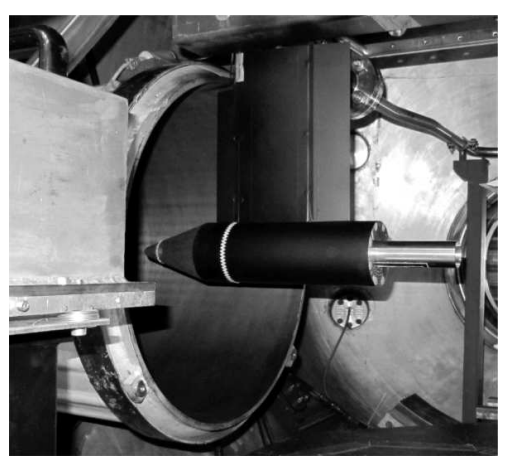

Figure 2 Wind tunnel model in H2K

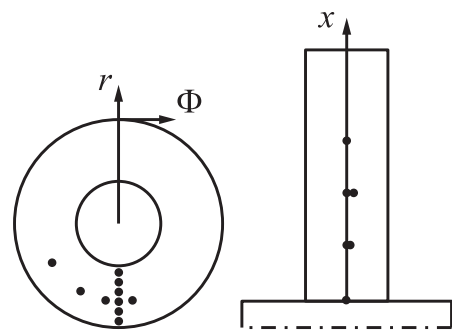

Figure 3 Sketch (rear and bottom views) of transducer positions
The H2K facility is a blowdown wind tunnel with a free jet test section (Fig. 2). Six different contoured and axially symmetric wind tunnel nozzles are available for aerodynamic testing at Mach 4.8, 5.3, 6.0, 7.0, 8.7, and 11.2. For aerothermal tests on probes, smaller nozzles with Mach numbers of 3.0 and 5.0 are also in use. The required pressure ratio for the high Mach numbers is made feasible due to the vacuum sphere downstream of the nozzle and diffuser. The air is preheated in order to prevent condensation of air and for tests at high temperatures. Eight electric heaters with a maximum electrical power of 5-megawatt heat are available to heat the air up to $1100 \mathrm{~K}$. The free-stream turbulence level of $\mathrm{H} 2 \mathrm{~K}$ is $0.8 \%$ to $0.9 \%$.

The wind tunnel model is equipped with flush-mounted unsteady pressure transducers on the base and on the surface of the nozzle that feature a measuring range of 0.35 bar. A sketch of the arrangement is given in Fig. 3. Throughout this paper, a particular attention will be paid to the signals given by unsteady transducers on the nozzle surface. Four

transducers are located $2,29,56$, and $83 \mathrm{~mm}$ downstream of the base on the strut-averted side at $\varphi=180^{\circ}$ with $\varphi$ being defined according to the used righthand coordinate system with the $x$-axis pointing out of the base plate. The two transducers left are placed at $(x, \varphi)=\left(29 \mathrm{~mm}, 185^{\circ}\right)$ and $\left(56 \mathrm{~mm}, 190^{\circ}\right)$. Nondimensionalized with the diameter of the model, this corresponds approximately to $x / D \sim 0,0.25,0.5$, and 0.75 . The pressure signal is acquired with a resolution of 24 bit and a sampling rate of $100 \mathrm{kHz}$ by using a NI PXIe-4331 bridge module. For each transducer, $2 \cdot 10^{6}$ samples are recorded per run.

\section{COMPUTATIONAL APPROACH}

The time-resolved numerical computations of the flow field around the generic rocket configuration are performed by the Institute of Aerodynamics at the 
$p / p_{\infty}$

$\begin{array}{lllllllllllll}0.1 & 0.2 & 0.3 & 0.4 & 0.5 & 0.6 & 0.7 & 0.8 & 0.9 & 1.0 & 1.1 & 1.2 & 1.3\end{array}$

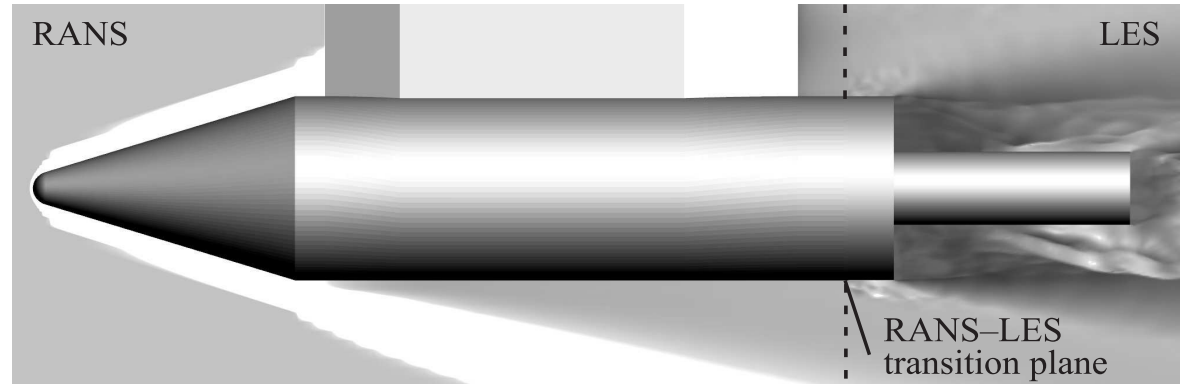

Figure 4 Flow field decomposition into RANS and LES zones

RWTH Aachen University using a zonal RANS/LES approach. The computation domain around the rocket configuration is split into a main body zone with an attached flow where the turbulent flow field is predicted by solving the RANS equations and a wake zone where the unsteady separated flow is time-resolving computed by the LES (Fig. 4).

\subsection{Flow Solver}

The computations are done on a structured vertex-centered multiblock grid using an in-house zonal RANS/LES finite volume flow solver. The Navier-Stokes equations of three-dimensional (3D) unsteady compressible flow are discretized in conservative form by a mixed centered upwind AUSM (advective upstream splitting method) scheme [17] at second-order accuracy for the Euler terms and by a second-order accurate centered approximation for the viscid terms accounting for low numerical dissipation. The temporal integration is performed by an explicit 5-stage Runge-Kutta method with second-order accuracy as well. The LES formulation is based on the monotone integrated LES (MILES) approach [18] modeling the impact of the subgrid scales by numerical dissipation. A detailed description of the fundamental LES solver is given by Meinke et al. [19] and its convincing solution quality for fully turbulent sub- and supersonic flows is discussed by Alkishriwi et al. [20] and El-Askary et al. [21]. The RANS part is based on the same overall discretization schemes and a one-equation turbulence model of Spalart and Almaras [22] to close the time-averaged equations.

\subsection{Reformulated Synthetic Turbulent Generation Method}

The challenging transition from the RANS to the LES zone is performed by a Reformulated Synthetic Turbulence Generation (RSTG) method developed 
by Roidl et al. $[23,24]$ that allows a reconstruction of the time-resolved turbulent fluctuations from the time-averaged upstream RANS solution. The RSTG method is based on the synthetic eddy method (SEM) of Jarrin et al. [25] and Pamiès et al. [26] and describes turbulence as a superposition of coherent structures. These structures are generated over the LES inlet plane by superimposing the influence of virtual eddy cores which are defined in a virtual volume $V_{\text {virt }}$ around the inlet plane that has the streamwise, wall-normal, and spanwise dimensions of the turbulent length scale $l_{x}$, the boundary-layer thickness at the inlet $\delta_{0}$, and the width of the computational domain $L_{z}$. The turbulent length scales that describe the spatial properties of the synthetic structure depend on the distance from the wall and are derived from the turbulent viscosity $\mu_{t}$ of the upstream RANS solution and scaled with the Reynolds number and the associated convection velocity. As a result, the final velocity signal is composed of an averaged velocity component $\overline{u_{i}}$ which is provided from the upstream RANS solution and the normalized stochastic fluctuations $u_{i}^{\prime}$ which are subjected to a Cholesky decomposition $a_{i, j}$ to assign the values of the Reynolds-stress tensor. The used RSTG method allows the transitional length to be reduced to only two boundary-layer thicknesses. For a detailed description of the applied zonal RANS/LES method including the shape functions and length scale distributions as well as its validation against pure LES and DNS data for compressible flows with separated boundary layers, the reader is referred to $[23,24]$.

\subsection{Computational Grid}

According to the applied zonal approach, the RANS domain covers the main rocket geometry and the LES region encompasses the wake. Since the main body geometry is identical for both considered cases, a modular setup is used for the computational grids that consists of using the same RANS mesh for the main body and two different LES meshes for the applied aft-body extensions. To illustrate the applied grid topologies, the meshes for the RANS and LES zones used for the configuration with nozzle dummy are shown in Figs. $5 a$ and $5 b$.

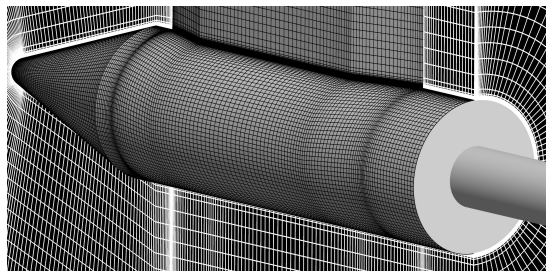

(a)

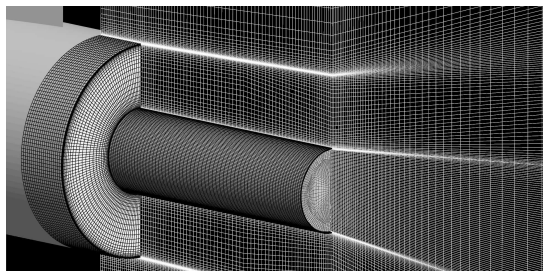

(b)

Figure 5 Computational grids for the RANS ( $a$ ) and LES (b) zones. Every 4th grid point is shown 
Table 2 Cell sizes in inner and outer coordinates for RANS and LES zones

\begin{tabular}{lccccc}
\hline Zone & $\Delta x / l^{+}$ & $\Delta r / l^{+}$ & $\Delta \varphi / l^{+}$ & $\Delta r / \delta$ & Grid points \\
\hline RANS & 60 & 0.7 & 60 & $1.7 \cdot 10^{-3}$ & $14.8 \cdot 10^{6}$ \\
LES & 30 & 0.7 & 20 & $1.7 \cdot 10^{-3}$ & $\approx 23 \cdot 10^{6}$ \\
\hline
\end{tabular}

To reduce computational costs, the grids span a region of $180^{\circ}$ with a mirror boundary condition in the symmetry plane. The RANS grid for the main body ranges from $-8 D$ to $0 D$ in the streamwise and up to $4 D$ in the radial direction with $D$ being the diameter of the cylindrical main body part and the origin of ordinates lying in the center of the rocket base. The RANS/LES transition plane is positioned at $x=-0.25 D$ upstream of the base shoulder with the boundary layer thickness being $\delta \sim 0.1 D$ which yields a satisfactory transition length of at least two boundary-layer thicknesses as expected by the RSTG method. The LES grid spans between $x=-0.2 D$ and $3.2 D$ in the streamwise and up to $3.6 D$ in the radial direction in the above-mentioned coordinate system. The maximum grid resolution for the RANS and LES zones in inner and outer coordinates with their total grid sizes is given in Table 2. In the initial stage of the computations, a coarser grid with a resolution of $y^{+}=2, x^{+}=60$, and $\varphi^{+}=40$ was used and the same wake flow topology including the shock positions and reattachment lengths (see subsection 5.1) was obtained as for the final grid. To enhance the capturing of smaller scales, a refined grid with the resolution of $y^{+}=0.7, x^{+}=30$, and $\varphi^{+}=20$ was used satisfying typical mesh requirements for LES given in [27].

\section{RESULTS}

The presented results are divided into three sections. First, a brief description of the flow along the main body including an estimate of the impact of the vertical strut needed for the wind tunnel tests is given in subsection 5.1. Additionally, the wake flow topologies of the investigated configurations are qualitatively described by means of experimental and numerical schlieren pictures illustrating the positions of the expansion and shock waves. Afterwards, in subsection 5.2, the distribution of the time-averaged and rms values of the wall pressure fluctuations along the base and nozzle dummy walls are compared to each other and discussed with respect to the detected flow patterns. A detailed spectral analysis of the detected dynamic behavior of the wake flow is presented in subsection 5.3.

\subsection{General Characterization of the Flow Field Topology}

To illustrate the flow field topology around the investigated generic rocket models, the instantaneous Mach number and wall pressure coefficient distributions 


\section{$\mathrm{Ma}$}

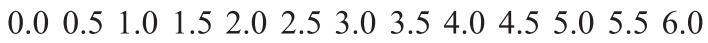

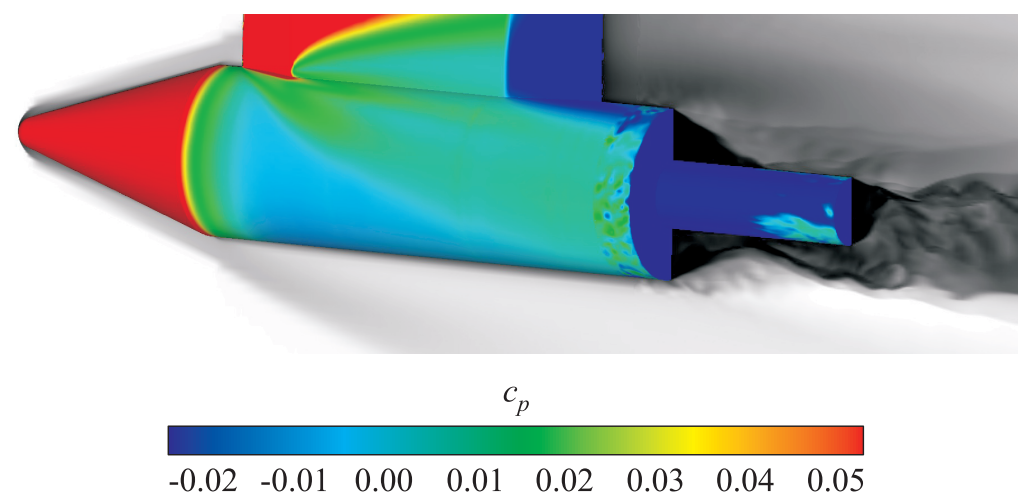

(a)

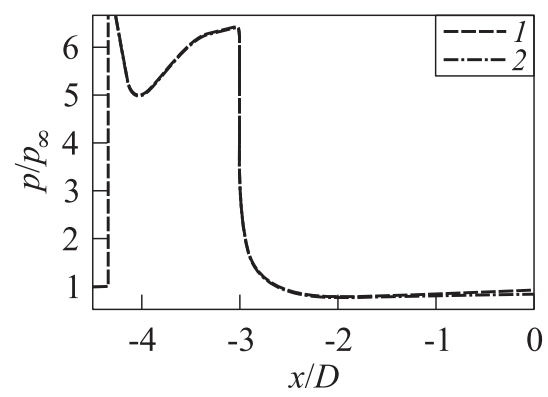

(b)

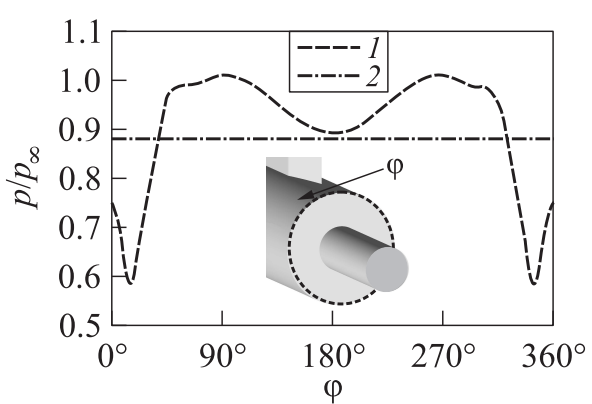

(c)

Figure 6 Configuration with nozzle dummy: (a) instantaneous Ma (black and white) and $c_{p}$ (color) distribution; $(b)$ pressure distribution along the main body on the strutaverted side compared to an axisymmetric case computed via RANS $(1$ - with support at $\varphi=180^{\circ}$; and 2 - without support); and (c) circumferential pressure distribution on the base shoulder compared to an axisymmetric case (LES results) (1 - with support; and 2 - without support)

are presented in Fig. $6 a$ for the configuration with a nozzle dummy. First, the incoming freestream with $\mathrm{Ma}_{\infty}=6.0$ is deflected at the tip parallel to the cone wall forming a detached bow shock, leading to an increase of the pressure as indicated in Fig. $6 b$ by the line plot of the pressure ratio along the model wall on the strut averted side. At the junction to the cylindrical part, the flow is redirected parallel to the free-stream by an expansion fan causing the pressure to decrease again (at $x / D=-3$ in Fig. $6 b$ ). On the upper side, a second shock and expansion wave system is formed around the double-wedge profiled strut which 


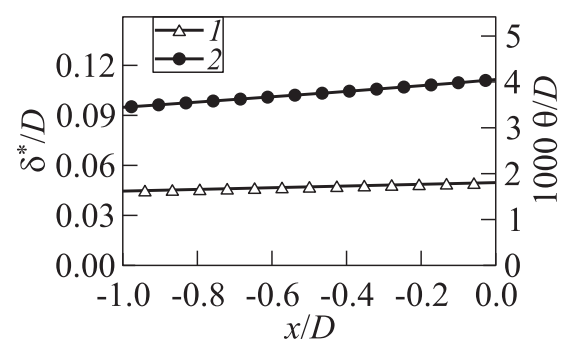

(a)

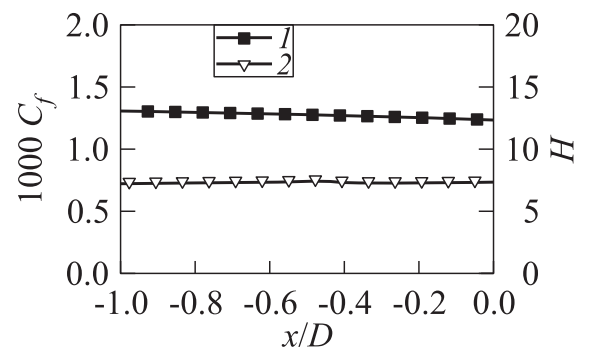

(b)

Figure 7 Incoming supersonic boundary layer on the strut averted side $\left(\varphi=180^{\circ}\right)$ : (a) displacement $\delta^{*}(1)$ and momentum $\theta(2)$ thicknesses; and (b) boundary-layer shape factor $H(1)$ and skin friction coefficient $C_{f}(2)$

subsequently interacts with the shock and expansion waves emanating from the main body. As a footprint of this interaction, the nonaxisymmetric distribution of the wall pressure coefficient shown in Fig. $6 a$ can be used to assess the impact of the vertical support on the flow field.

The comparison of the azimuthal distribution of the wall pressure along the base shoulder to that of an axisymmetric case shown in Fig. $6 c$ indicates that the influence of the vertical support is nonnegligible along the whole circumference. Although on the strut averted side the deviation is minimal, the performed numerical simulations incorporate the vertical support from the experimental investigations in order to allow a better comparison and validation of the obtained results. As shown in Fig. $7 a$, on the strut-averted side $\left(\varphi=180^{\circ}\right)$ near the RANS-LES transition region $(x / D=-0.25)$, the incoming supersonic boundary layer possesses a displacement thickness of about $\delta^{*} \sim 0.05$ and a momentum thickness of $\theta \sim 0.004 D$ having a total boundary layer thickness of $\delta \sim 0.1 D$. As expected for supersonic boundary layers at high Mach numbers [28], the corresponding shape factor (Fig. $7 b)$ is significantly higher $(H=\theta / \delta \sim 12)$ compared to typical values of subsonic equilibrium turbulent boundary layers that are usually in the range of $H \sim 1.5$.

Besides the nonaxisymmetric pressure distribution due to the interaction between shock and expansion wave systems of the main body and the strut discussed previously, the strut wake itself possesses a particularly low specific momentum $\left(\left.\rho u\right|_{\phi=0^{\circ}} /\left.\rho u\right|_{\phi=180^{\circ}}=0.14\right.$ outside of the main body boundary layer). This results in a stronger deflection of the weakened flow towards the model $x / D$-axis on the strut side downstream of the expansion on the base shoulder, which leads to an earlier and more pronounced reattachment compared to the rest of the circumference and to a nonsymmetrical flow pattern in the wake, as indicated in Fig. 8 showing the instantaneous Mach number distribution in the base region of the investigated configurations. 


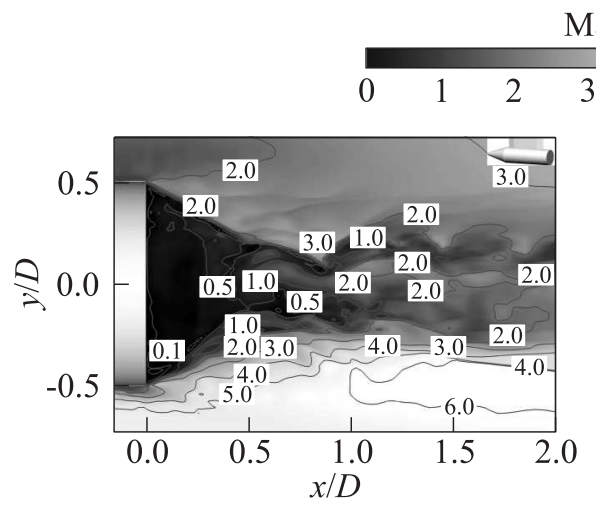

(a)

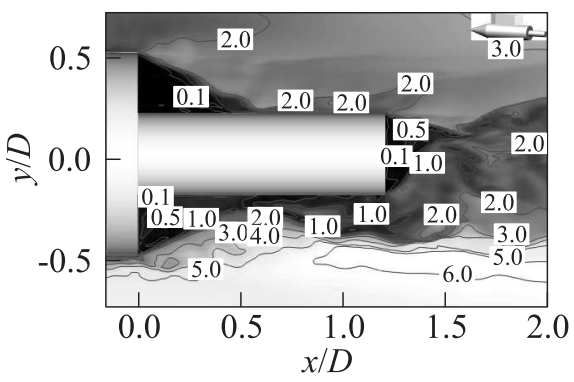

(b)

Figure 8 Instantaneous Mach number distribution in the wake of the configurations in the symmetry plane: $(a)$ blunt base; and $(b)$ nozzle dummy

The wake flow at $\mathrm{Ma}_{\infty}=6$ is determined by strong compressibility effects. To characterize the wake flow topology of the investigated configurations, the exponentially weighted density gradient computed according to [29] is used allowing to better identify the boundary and shear layers, expansion, and shock waves. The obtained instantaneous numerical schlieren pictures are presented in Fig. 9. Note that the incoming boundary layer and the wake flow topologies are asymmetrical with regard to the horizontal plane due to the vertical strut support attached to the forebody, which is indicated by the anotation "wake

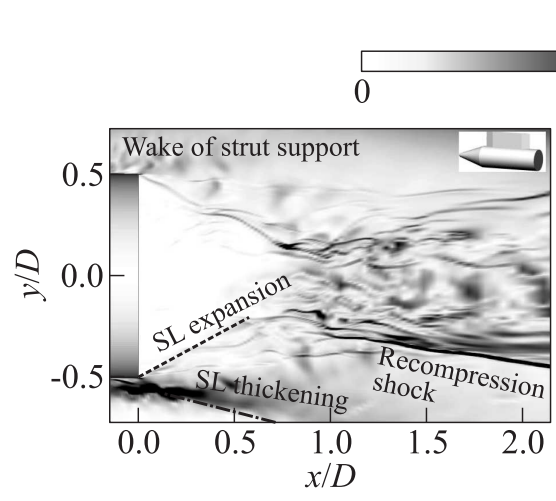

(a)

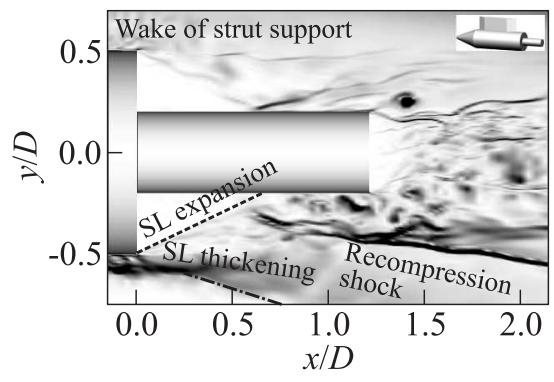

(b)

Figure 9 Instantaneous numerical schlieren pictures in the wake of the configurations in the symmetry plane [13]: (a) blunt base; and (b) nozzle dummy 


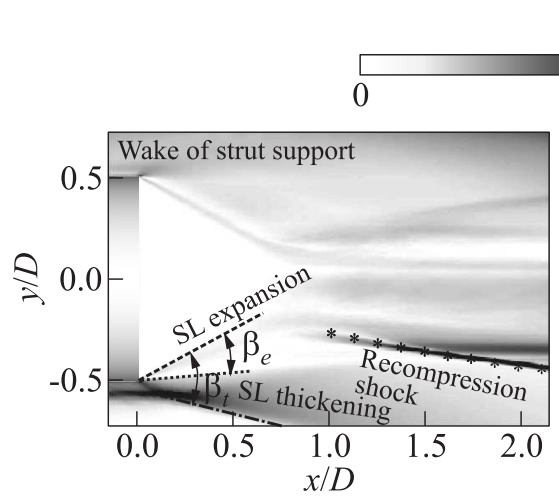

(a)
$\Delta \hat{\rho}$

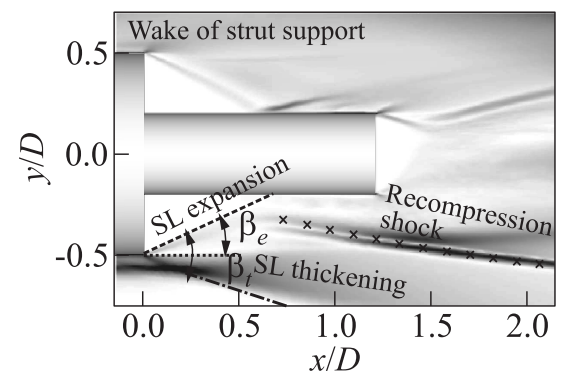

(b)

Figure 10 Time-averaged numerical schlieren pictures in the wake of the configurations in the symmetry plane. The positions of the recompression shocks detected in the experiments are indicated by stars (blunt base) $(a)$ and crosses (nozzle dummy) (b)

of strut support" in the upper left corners of Figs. $9 a$ and $9 b$. Obviously, the wakes of the investigated configurations are first and foremost characterized by the expansion of the supersonic shear layers shed from the base shoulder associated with a strong radial deflection towards the models longitudinal axes (annotated as "SL expansion" in Fig. 9). Further downstream of the base, the shed shear layers subsequently interact with either the recirculating flow in the blunt-base case or the solid surface in the nozzle-dummy case, which is accompanied by the formation of the recompression shocks correspondingly marked in the bottom right corners of Figs. $9 a$ and $9 b$. As a result, the confined lowpressure separation bubbles containing large-scale recirculation vortices form at the base. Therefore, the wakes of both configurations feature a pronounced unsteady behavior that is additionally excited by the unstable shedding shear layer undergoing turbulent mixing (annotated as "SL thickening" in the bottom left corners of Figs. $9 a$ and $9 b$ ).

Figure 10 shows the time-averaged numerical schlieren pictures allowing a general characterization of the mean wake flow topologies and a comparison with the experimental data. In the blunt base case shown in Fig. 10a, the expansion and following free reattachment of the shedding shear layer at $x / D=0.75$ causes the formation of a single recirculation zone with, as will be analyzed in detail in subsection 5.2, a nearly constant base pressure level. The effect of the nozzle dummy is to replace the pure wake structure by a free shear layer interacting with a surface such that a wall-bounded shear layer develops. The flow reattaches on the wall at about $x / D=0.6$ and the second smaller recirculation region appears at the rear end of the nozzle dummy as shown in Fig. 10b. Note 
that the mean shear layer expansion angle $\beta_{s}$ is approximately alike for both afterbodies $\left(\beta_{e} \approx 26^{\circ}\right)$, which indicates a nearly equal base pressure level being proved in subsection 5.2. Finally, to validate the numerically computed flow field topology, the time-averaged positions of the recompression shocks detected experimentally by Saile $e t$ al. [16] using high-speed schlieren measurements, an example of which is shown in Fig. 11 for the

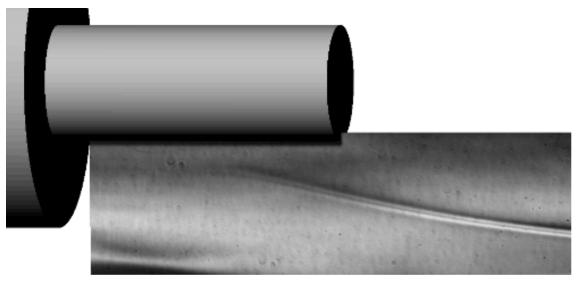

Figure 11 Standard deviation of a sequence of high-speed schlieren images of the recompression shock exemplary for the dummy nozzle configuration [16] nozzle dummy configuration, are drawn in Fig. 10 (indicated by stars for the blunt base and by crosses for the nozzle dummy configuration) and illustrate a good agreement between the numerical and the experimental results for both configurations.

\subsection{Spatial Wall Pressure Distribution}

As was shown in the previous subsection, the base regions of the investigated configurations are determined by separating and reattaching shear layers accompanied by recompression shocks, expansion waves, and recirculation bubbles. Statnikov et al. [13] showed that the strength of the recompression shocks and expansion waves directly depends on the deflection of the shedding shear layer which is determined by the tail geometry and, possibly, other geometrical obstacles, e.g., a strongly aftexpanding jet plume that emanates from the nozzle in real flight conditions. For the blunt-base and nozzle-dummy configurations, the large expansion angles $\beta_{e}$ of the shedding shear layer shown in Fig. 10 result in a strong base pressure drop compared to the mean pressure level of the incoming supersonic boundary layer on the main body. This fact is illustrated in Fig. $12 a$ showing the time-averaged pressure levels for the base regions of both configurations and the incoming boundary layer at $x / D=-0.1$ upstream of the base extracted from the LES data. The comparison of three curves illustrates:

- a strong pressure drop of a similar magnitude due to the nearly equal mean shear layer deflection angles shown in Fig. $10\left(\beta_{e} \approx 26.5^{\circ}\right.$ for the blunt-base case and $\beta_{e} \approx 25.5^{\circ}$ for the nozzle-dummy configuration);

- a slight increase of the mean base pressure value from $p / p_{\infty} \approx 0.2$ for the blunt-base to $p / p_{\infty} \approx 0.25$ for the nozzle-dummy configuration; and

- a significantly reduced but still present asymmetrical effect of the strut support in the azimuthal direction. 


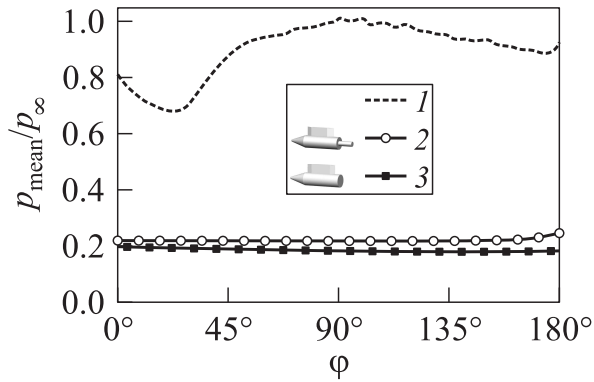

(a)

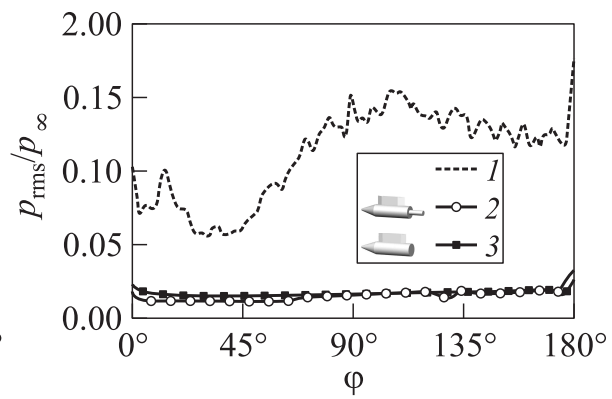

(b)

Figure 12 Comparison of the mean $(a)$ and $\mathrm{rms}(b)$ values of the pressure oscillations on the base wall and in the boundary layer along the main body (extracted from [13]): 1 - main body at $r / D=0.5$ and $x / D=-0.1$; and 2 - nozzle dummy and 3 - blunt base at $r / D=0.4, x / D=0$

The detected small rise of the time-averaged base pressure level due to the nozzle dummy is accompanied by a minimal increase of the rms values of the base pressure fluctuations only on the strut-averted side shown in Fig. $12 b$ which are about $p_{\text {rms }}^{\prime} / p_{\infty} \approx 0.02$ for the blunt-base and $p_{\text {rms }}^{\prime} / p_{\infty} \approx 0.025$ for the nozzle-dummy configurations corresponding to about $10 \%$ of the local mean value. Particularly worth mentioning is the fact that compared with the pressure fluctuations on the main body at $x / D=-0.1$ (curve 1 in Fig. 12b), the absolute amplitudes of the pressure oscillation on the base wall are considerably lower than in the incoming turbulent supersonic boundary layer with its $p_{\mathrm{rms}}^{\prime} / p_{\infty} \approx 0.05-$ 0.15 .

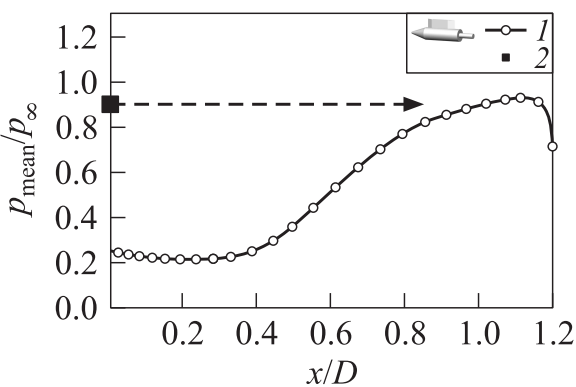

(a)

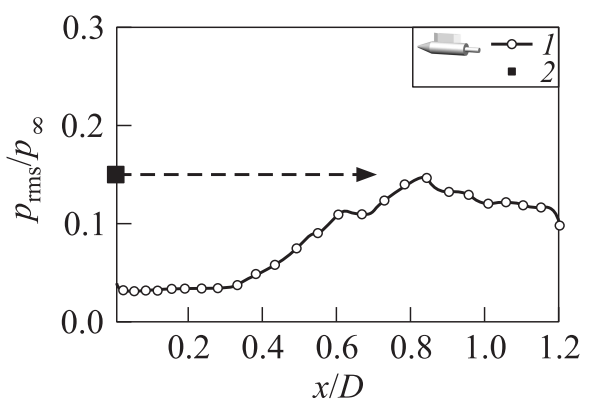

(b)

Figure 13 Comparison of the mean $(a)$ and rms $(b)$ values of the pressure oscillations on the nozzle dummy and in the boundary layer along the main body (extracted from [13]): 1 - nozzle dummy $\left(\varphi=180^{\circ}\right)$; and $2-$ main body $(r / D=0.5$, $x / D=-0.1$, and $\left.\varphi=180^{\circ}\right)$ 
However, the wall pressure mean and oscillatory values are significantly higher when considering the nozzle wall as shown in Fig. 13. Due to a solid reattachment, the rms values for the nozzle dummy configuration reach up to $p_{\text {rms }}^{\prime} / p_{\infty}$ $\approx 0.15$, i. e., practically up to the corresponding value of the pressure fluctuations of the incoming supersonic boundary layer indicated by the dashed line in Fig. 13b. The same trend also applies for the mean pressure level which increases up to $p / p_{\infty} \approx 1$ downstream of the reattachment point (see Fig. 13a). The observed similar mean and rms values of the wall pressure signal indicate that the pressure perturbations on the nozzle dummy and the incoming boundary layer are the related phenomena which are analyzed in the next subsection.

\subsection{Analysis of the Dynamic Wake Flow Behavior}

To analyze the assumed connection between the pressure perturbations on the nozzle dummy and the incoming boundary layer, the LES results are particularly useful thanks to their high spatial and temporal resolution. Figure $14 a$ visualizes the instantaneous vortex structures in the wake region for the nozzle-dummy configuration by means of $\lambda_{2}$-contours [30] coloured by the Mach number. It can be seen that compared to the boundary layer, when shedding from the base shoulder, the coherent structures in the shear layer start increasing in size and merge with neighboring structures (region (ii) in Fig. 14a), which is similar to the formation of Brown-Roshko-like structures [31,32] due to a density gradient between the outer flow and the recirculation area. Note that this fact was already

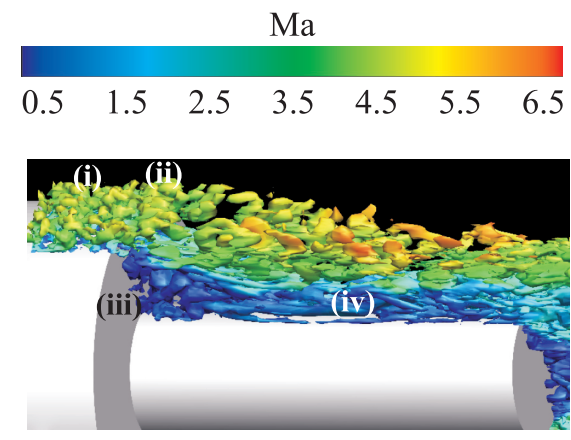

(a)

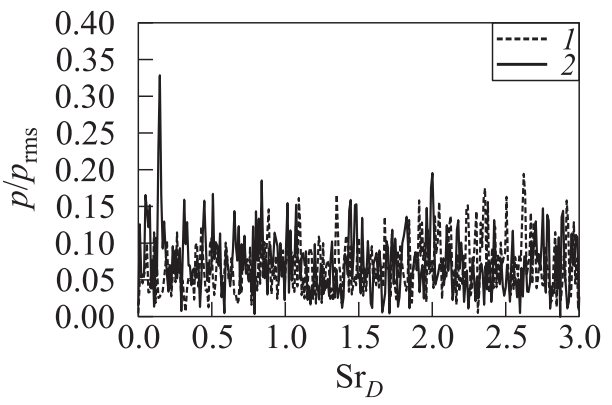

(b)

Figure 14 Large-eddy simulation: analysis of the boundary and shear layer instabilities for the nozzle dummy configuration: $(a) \lambda_{2}$ iso-contour coloured by Mach number; white numbers indicate region of interest; and $(b)$ pressure spectra in the boundary $\left(1-x / D=-0.1, r / D=0.5, \varphi=180^{\circ}\right)$ and shear $(2-x / D=0.1, r / D=0.48$, $\varphi=180^{\circ}$ ) layers 


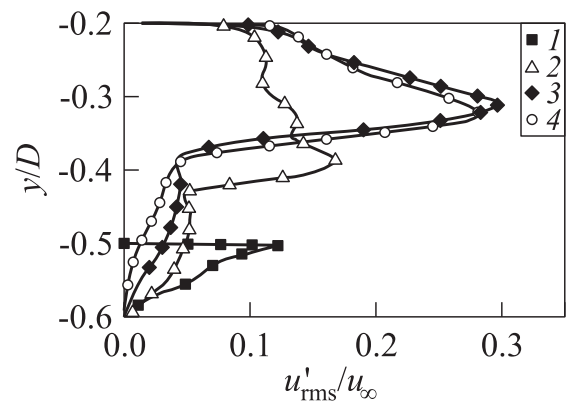

(a)

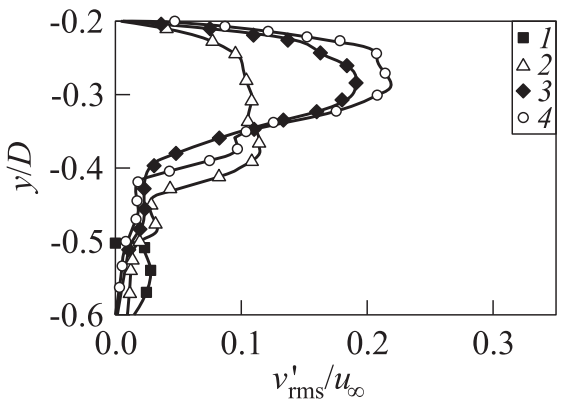

(b)

Figure 15 Wall-normal distribution of the axial $(a)$ and radial $(b)$ velocity fluctuations in the wake of the nozzle-dummy configuration for several axial positions on the strut-averted side $\left(\varphi=180^{\circ}\right): 1-x / D=-0.05 ; 2-0.25 ; 3-0.5 ;$ and $4-$ $x / D=0.75$

seen in the context of the thickening of the shedding shear layer qualitatively observed in Fig. 9 in subsection 5.1. To quantitatively discuss the shear layer instability process, Fig. 15 shows the wall-normal distribution of the rms values of the axial and radial velocity fluctuations for several axial positions for the nozzle dummy configuration on the strut-averted side $\left(\varphi=180^{\circ}\right)$. Slightly upstream of the model shoulder $(x / D=-0.05)$, the incoming supersonic boundary layer features a typical distribution of the normal Reynolds shear stresses (curve 1) with $u_{\text {rms }}^{\prime} / u_{\infty} \sim 0.1$ and $\sim 0.03$. Downstream of the separation of the boundary layer, the velocity fluctuations rapidly increase when moving downstream of the base illustrating the spatial evolution of the unstable shear layer. The axial velocity fluctuations (Fig. 15a) increase by about a factor of $\approx 2.5$, when moving in the axial direction along the specified distance, reaching up to $u_{\mathrm{rms}}^{\prime} \approx 0.3 u_{\infty}$. Due to the turbulent mixing process, the rms values of the radial velocity fluctuations (Fig. $15 b$ ) grow even more significantly, i. e., from initially $\approx 3 \%$ up to $\approx 20 \%$ of $u_{\infty}$. When moving in the $x$-direction, the ordinates of the maximum velocity fluctuations also clearly shift towards $y / D=-0.2$, i.e., the wall of the nozzle dummy, indicating the strong radial deflection of the shear layer associated with the expansion on the model shoulder. Furthermore, the radial extent of the regions of high fluctuation levels becomes larger when moving in the $x$-direction as a result of the growth of the shear-layer thickness.

The boundary layer separation on the model shoulder also changes the frequency content of its pressure signal. As indicated by curve 2 in Fig. 14b, the pressure fluctuations inside the shed shear layer (region (ii) in Fig. 14a) feature a sharp peak at a distinct dominant frequency of about $\operatorname{Sr}_{D}=0.2$ that appears due to the vortex shedding at the model shoulder [33] and originally 


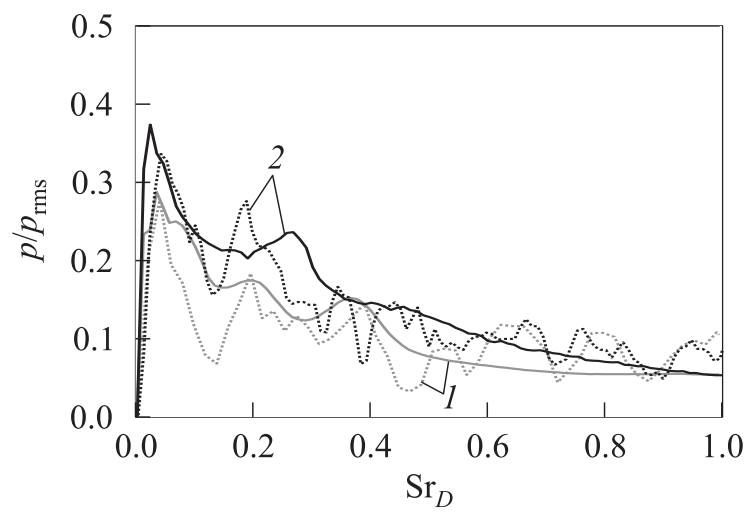

Figure 16 Comparison of the numerical RANS/LES (dashed curves) and experimental (solid curves) pressure spectra on the base wall (extracted from [13]) at $\varphi=180^{\circ}$ and $r=40 \mathrm{~mm}\left(t=1200 \cdot 50 D / U_{\infty}\right): 1$ - blunt base; and 2 - nozzle dummy

is not present in the incoming boundary layer. The latter possesses a broadband spectrum without any distinct dominant frequencies over a wide range of Strouhal numbers, as illustrated by curve 1 in Fig. $14 b$.

Regarding the base region (zone (iii) in Fig. 14a) that is directly influenced by the vortex shedding process on the rocket shoulder, dominant peaks around $\mathrm{Sr}_{D} \approx 0.2-0.27$ also clearly appear in the corresponding spectra of the wall pressure fluctuations for both experimental and numerical investigation techniques in the blunt-base and nozzle-dummy cases as shown in Fig. 16. Additionally, the numerical and experimental base pressure spectra feature also clearly pronounced peaks in the range of $\mathrm{Sr}_{D} \approx 0.05$ which are presumably associated with the oscillatory movement of the large-scale toroidal vortex within the separation bubble as known from the former investigations performed by the authors on a similar generic configuration [34].

The dynamic behavior changes crucially when moving downstream along the nozzle dummy as indicated in Fig. 17 showing the spectra of the pressure fluctuations at different axial positions on the wall of the nozzle dummy. First, it can be noted that, compared to their LES counterparts shown in Fig. $17 a$, the spectra in Fig. $17 b$ obtained from the experiment are smoother due to significantly longer wind tunnel runs at the H2K facility and, consequently, superior signal lengths improving the statistical quality of the spectral analysis compared to the simulations. The time-resolved zonal RANS/LES data are analyzed over a time interval of $50 t_{\text {ref }}$ with $1 t_{\text {ref }}$ being the reference time unit needed by a particle moving with the free-stream velocity $u_{\infty}$ to cover one reference length equal to the main body diameter $D$ of the launcher. The data are sampled with $0.1 t_{\text {ref }}$ that corresponds to a sampling frequency of $\mathrm{Sr}_{D}=10$. According to the Nyquist 


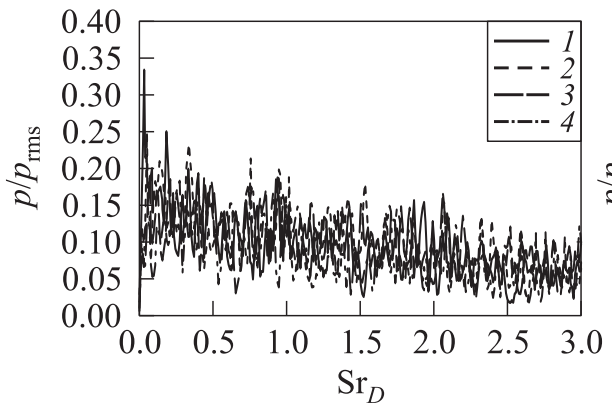

(a)

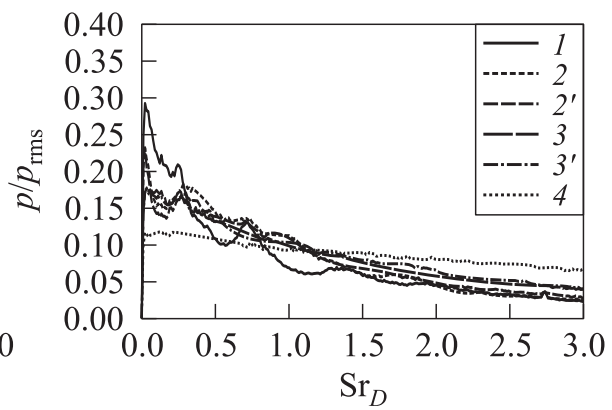

(b)

Figure 17 Wall pressure spectra along the nozzle dummy at $x / D=0.25$ and $\varphi$ $=180^{\circ}(1) ; x / D=0.25$ and $\varphi=180^{\circ}(2)$ and $185^{\circ}\left(2^{\prime}\right) ; x / D=0.5$ and $\varphi=180^{\circ}$ (3) and $190^{\circ}\left(3^{\prime}\right)$; and $x / D=0.75$ and $\varphi=180^{\circ}(4)$ : (a) LES; and (b) experiments

criterion, the available LES data sets allow to capture the frequencies in the range of $0.02 \leqslant \operatorname{Sr}_{D} \leqslant 5$. The analyzed time interval of the performed wind tunnel runs is $7.1 \mathrm{~s}$ which leads to 1200 times longer experimental signals and more reliable spectral analyses. While in the base area the available length of the LES data of $50 t_{\text {ref }}$ is satisfactory as was shown in Fig. 16, it becomes more critical in a statistical sense when moving downstream of the base area into the region near the reattachment point. Unlike the near-wake, this region is strongly affected by the variety of involved turbulent length and time scales determined by the boundary layer. This fact results in rather wide peaks in the experimental spectra as can be observed in Fig. $17 b$ showing the Fourier transforms of 1200 times longer wall pressure signals which were measured at several discrete positions on the nozzle dummy in the experiment.

On the whole, the presented wall pressure spectra along the nozzle dummy show that the signals in the corner at $x / D=0.0$ are consistent with the finding on the fluctuations on the base seen in Fig. 16, meaning periodic oscillations in the low-frequency range at about $\mathrm{Sr}_{D} \approx 0.05$ and $\approx 0.2-0.27$. Additionally, another higher dominant frequency can be found, which centers at $\operatorname{Sr}_{D} \approx 0.7$. Especially, the oscillations taking place at 0.05 and 0.27 can also be found for the two other transducers in the recirculation region but the frequencies are less distinct and widened over a larger range.

The sensors at $x / D=0.0,0.25$, and 0.5 still indicate periodic mechanisms inside the recirculation region which, however, become less dominant when moving downstream to the zone (iv) in Fig. $14 a$ where the shed shear layer reattaches on the nozzle dummy. The spectrum of the signal at $x / D=0.75$ reveals that the region around the reattachment point exhibits no distinct frequencies and can be rather characterized as broadband, exactly as it was the case for the incoming 
boundary layer previously discussed in the context of Fig. 14b. This fact is also in agreement with the observation made in subsection 5.2 that the mean wall pressure and the rms value of its fluctuations grow continuously along the nozzle dummy up to their maxima near the reattachment point where they reach the values of the incoming boundary layer.

\section{CONCLUDING REMARKS}

The nature of the turbulent wake flow of a generic space transportation system in the hypersonic flow regime at Mach 6 has been investigated experimentally by means of high-speed schlieren images and unsteady pressure measurements as well as numerically using a time-resolving zonal RANS/LES method. To analyze the influence of the nozzle extension on the base flow field, two different aft-body geometries consisting of a blunt base and an attached cylindrical nozzle dummy are considered. After an introductory description of the flow topology, the experimental and numerical results are compared to each other with regard to the steady-state and dynamic wake flow characteristic to validate the applied methods and to justify the detected phenomena.

The investigations to the main body reveal the 3D nature of the incoming flow due to the strut support of the wind tunnel model. The flow field around the main body is dominated by the interaction of two systems of shocks and expansion waves, which arise on the axisymmetric rocket body and the vertical strut support, and differs from the axisymmetric case. In addition, the wake of the strut itself possesses a noteworthy lower specific momentum than that on the opposite circumferential positions and, consequently, causes a further nonaxisymmetric impact on the base flow of the rocket models. Nevertheless, on the strut-averted side near the base region, the three-dimensionality is not as prominent as upstream of the base, meaning the investigations with the strut support are justified.

The wake regions of the investigated configurations are dominated by separating and reattaching shear layers accompanied by recompression shocks, expansion waves, and recirculation bubbles. A good agreement between the numerical and experimental results is found with respect to the wake flow topology: the positions of the reattachment shocks in the wakes of both configurations coincide for both approaches. Depending on the applied aft-body extensions, the timeaveraged base pressure level, which constitutes a major part of the base drag, ranges from $p / p_{\infty} \approx 0.2$ for the blunt-base to $p / p_{\infty} \approx 0.25$ for the nozzle-dummy configuration. The rms values of the base pressure fluctuations were found to be about $10 \%$ of the local mean values for both investigated configurations and, consequently, are considerably lower compared to the pressure oscillations in the incoming turbulent hypersonic boundary layer. However, the wall pressure oscillations are significantly higher when considering the nozzle-dummy wall. Due to 
a solid reattachment, the corresponding rms values for the nozzle-dummy configuration reach up to $p_{\text {rms }}^{\prime} / p_{\infty} \approx 0.15$, which is caused by turbulent structures stemming from the incoming boundary and subsequent shed shear layer.

The presented spectral analyses of the wall pressure oscillations show a good agreement between the experimental and numerical results. The wall pressure oscillations slightly downstream of the reattachment region show rather a broadband spectrum with no distinct frequencies, which is determined by the incoming boundary and subsequent shed shear layer. On the other hand, the recirculation regions feature a pronounced periodic behavior which becomes more prominent closer to the base. The spectral analysis of the experimental and numerical wall pressure signals on the base returns dominant peaks at about $\operatorname{Sr}_{D} \approx 0.05$ and 0.2 for the blunt-body and $\mathrm{Sr}_{D} \approx 0.05$ and $0.2-0.27$ for the nozzle-dummy configurations. The frequencies around $\mathrm{Sr}_{D} \approx 0.2-0.27$ are attributed to the shear-layer instability, while the lower-frequency modes are referred to the inner dynamics of the recirculation bubbles.

Future investigations of the wake flow physics of space launchers will be performed on configurations with jets and extended to helium nozzle flows to better model the conditions in the wake of a rocket launcher. Besides that, a particular focus of the future analyses will lie on the transonic flight regime where the structure loads reach the maximum and the phenomenon of buffeting occurs. A side-by-side combination of the presented experimental and numerical investigation techniques will allow to gain a better insight into the wake flow physics and develop new methods for its efficient control.

\section{ACKNOWLEDGMENTS}

Financial support from the German Research Foundation (Deutsche Forschungsgemeinschaft - DFG) in the framework of the Sonderforschungsbereich Transregio 40 is gratefully acknowledged by the authors. Computational resources have been provided by the Stuttgart High-Performance Computing Center (HLRS).

\section{REFERENCES}

1. Rollstin, L. R. 1987. Measurement of in-flight base pressure on an artillery-fired projectile. AIAA Paper No. 87-2427.

2. Mathur, T., and J.C. Dutton. 1996. Base-bleed experiments with a cylindrical afterbody in supersonic flow. J. Spacecraft Rockets 33(1):30-37.

3. Bannink, W., E. Houtman, and P. Bakker. 1998. Base flow underexpanded exhaust plume interaction in a supersonic external flow. AIAA Paper No. 98-1598.

4. Scarano, F., B. W. van Oudheusden, W. J. Bannink, and M. Bsibsi. 2004. Experimental investigation of supersonic base flow plume interaction by means of particle 
image velocimetry. 5th Symposium (European) on Aerothermodynamics for Space Vehicles Proceedings. Cologne, Germany. ESA SP-563. 601-607.

5. Benay, R., and P. Servel. 2001. Two-equation $k-\sigma$ turbulence model: Application to a supersonic base flow. AIAA J. 39:407-416.

6. Papp, J., and K. Ghia. 2001. Application of the RNG turbulence model to the simulation of axisymmetric supersonic separated base flows. AIAA Paper No. 2001027.

7. Forsythe, J., K. Hoffmann, R. Cummings, and K. Squires. 2002. Detached-eddy simulation with compressibility corrections applied to a supersonic axisymmetric base flow. J. Fluid Eng. 124:911-923.

8. Kawai, S., and K. Fujii. 2004. Computational study of a supersonic base flow using LES/RANS hybrid methodology. AIAA Paper No. 2004-68.

9. Fureby, C., and K. Kupiainen. 2003. Large-eddy simulation of supersonic axisymmetric baseflow. Turbulent Shear Flow Phenomena (TSFP3). Japan.

10. Sandberg, R., and H. Fasel. 2004. High-accuracy DNS of supersonic base flows and control of the near wake. Users Group Conference Proceedings. IEEE Computer Society. $96-104$.

11. Sandberg, R., and H. Fasel. 2006. Numerical investigation of transitional supersonic axisymmetric wakes. J. Fluid Mech. 563:1-41.

12. Deck, S., and P. Thorigny. 2007. Unsteadiness of an axisymmetric separatingreattaching flow: Numerical investigation. Phys. Fluids 19:065103.

13. Statnikov, V., J.-H. Meiß, M. Meinke, and W. Schröder. 2013. Investigation of the turbulent wake flow of generic launcher configurations via a zonal RANS/LES method. CEAS Space J. 5(1-2):75-86.

14. Sippel, M., and A. Herbertz. 2008. System requirements on investigation of base flow/plume interaction. Notes of numerical fluid mechanics and multidisciplinary design. RESPACE-Key Technologies for Reusable Space Systems, results of a Virtual Institute Programme of the German Helmholts-Association (2003-2007). Springer. 98:3-19.

15. Murthy, A. V. 1974. Hypersonic flow over blunted slender wedges. J. Aircraft 11:649-651.

16. Saile, D., A. Gülhan, and A. Henckels. 2011. Investigations on the near-wake region of a generic space launcher geometry. AIAA Paper No. 2011-2352.

17. Liou, M.-S., and C. J. Steffen. 1993. A new flux splitting scheme. J. Comput. Phys. 107:23-39.

18. Boris, J., F. Grinstein, E. Oran, and R. Kolbe. 1992. New insights into large eddy simulation. Fluid Dyn. Res. 10:199-228.

19. Meinke, M., W. Schröder, E. Krause, and T. Rister. 2002. A comparison of secondand sixth-order methods for large-eddy simulations. Comput. Fluids 31:695-718.

20. Alkishriwi, N., M. Meinke, and W. Schröder. 2006. A large-eddy simulation method for low Mach number flows using preconditioning and multigrid. Comput. Fluids 35(10):1126-1136.

21. El-Askary, W., W. Schröder, and M. Meinke. 2003. LES of compressible wallbounded flows. AIAA Paper No. 2003-3554. 
22. Spalart, P., and S. Allmaras. 1992. A one-equation turbulence model for aerodynamic flows. AIAA Paper No. 92-0439.

23. Roidl, B., M. Meinke, and W. Schröder. 2013. Reformulated synthetic turbulence generation method for a zonal RANS-LES method and its application to zeropressure gradient boundary layers. Int. J. Heat Fluid Flow 44:28-40.

24. Roidl, B., M. Meinke, and W. Schröder. 2014. Boundary layers affected by different pressure gradients investigated computationally by a zonal RANS-LES method. Int. J. Heat Fluid Flow 45:1-13.

25. Jarrin, N., N. Benhamadouche, S. Laurence, and D. Prosser. 2006. A syntheticeddy-method for generating inflow conditions for large-eddy simulations. Int. J. Heat Fluid Flow 27:585-593.

26. Pamiès, M., P. Weiss, E. Garnier, S. Deck, and P. Sagaut. 2009. Generation of synthetic turbulent inflow data for large eddy simulation of spatially evolving wallbounded flows. Phys. Fluids 21:045103.

27. Piomelli, U., and E. Balaras. 2002. Wall-layer models for large-eddy simulations. Annu. Rev. Fluid Mech. 34:349-374.

28. Maeder, T., N.A. Adams, and L. Kleiser. 2001. Direct simulation of turbulent supersonic boundary layers by an extended temporal approach. J. Fluid Mech. 429:187-216.

29. Priebe, S., M. Wu, and P. Martin. 2009. Direct numerical simulation of a reflected-shock-wave/turbulent-boundary-layer interaction. AIAA J. 47(5):11731185. http://arc.aiaa.org/doi/abs/10.2514/1.38821.

30. Jeong. J., and F. Hussain. 1995. On the identification of a vortex. J. Fluid Mech. 285:69-94.

31. Brown, G. L., and A. Roshko. 1974. On density effects and large structure in turbulent mixing layers. J. Fluid Mech. 64:775-816.

32. Papamoschou, D., and A. Roshko. 1988. The compressible turbulent shear layer: An experimental study. J. Fluid Mech. 197:453-477.

33. Roshko, A. 1954. On the drag and shedding frequency of two-dimensional bluff bodies. NACA Technical Note 3169.

34. Saile, D., A. Gülhan, A. Henckels, C. Glatzer, V. Statnikov, and M. Meinke. 2013. Investigations on the turbulent wake of a generic space launcher geometry in the hypersonic flow regime. Progress in flight physics. Eds. Ph. Reijasse, D. D. Knight, M. S. Ivanov, and I. I. Lipatov. EUCASS advances in aerospace sciences book ser. EDP Sciences-TORUS PRESS. 5:209-234. 